\title{
On the Mechanism of Fermion-Boson Transformation
}

\author{
Andrzej Okniński
}

Received: 22 August 2013 / Accepted: 18 February 2014 / Published online: 2 May 2014

(C) The Author(s) 2014. This article is published with open access at Springerlink.com

\begin{abstract}
We study a fermion-boson transformation. Our approach is based on the $3 \times 3$ equations which are subequations of both the Dirac and Duffin-Kemmer-Petiau equations and thus provide a link between these equations. We show that solutions of the free Dirac equation can be converted to solutions of spin-0 Duffin-Kemmer-Petiau equation and vice versa. Mechanism of this transition assumes existence of a constant spinor.
\end{abstract}

Keywords Relativistic wave equations · Fermion-boson duality $\cdot$ Melosh-Wigner rotation

\section{Introduction}

Supersymmetry is a promising candidate for physics beyond the standard model and has the remarkable feature of mixing bosons and fermions. Recently, several supersymmetric equations related mainly to anyons in $(2+1)$ dimensions [1-5] as well as to the $(3+1)$ dimensional Majorana-Dirac-Staunton framework [6], uniting fermionic and bosonic fields, have been studied. However, while supersymmetry predicts existence of supersymmetric partners of known elementary particles, LHC experiments put their existence into doubt. We would like to propose an alternative view of fermion-boson mixing based on the FermiBose (FB) duality. The FB duality was first discovered for zero-mass relativistic equations: it was shown that some zero-mas equations, for example the massless Dirac equation, can describe fermionic as well as bosonic states [7-11], see also [12]. Furthermore, Polyakov discovered possibility of fermion-boson transmutation of elementary excitations of a scalar field interacting with the topological Chern-Simons term in $(2+1)$ dimensions [13]. The FB duality was further studied for the massive Dirac equation [14, 15] (earlier results in this direction, although limited to the stationary case, were published in Refs. [16, 17]).

In the present paper we attempt to find a fermion-boson transformation connecting solutions of the Dirac and spin-0 Duffin-Kemmer-Petiau (DKP) equations. Our approach is

\footnotetext{
A. Okniński $(\square)$

Politechnika Świętokrzyska, A1. 1000-lecia PP 7, 25-314 Kielce, Poland

e-mail: fizao@tu.kielce.pl
} 
based on the $3 \times 3$ equations, reviewed in the next Section, which are subequations of both the Dirac and DKP equations [18] (see also [19] for the interacting case) and thus provide a link between these equations. We interpret the $3 \times 3$ equations in Section 3 , showing that they can be transformed nonlocally into the form which can be obtained from the Dirac equation by application of the unitary Melosh transformation. In the next Section we demonstrate that, indeed, solutions of the free Dirac equation can be converted into solutions of the free DKP equation and vice versa. Mechanism of this transition assumes existence of an auxiliary constant spinor. We discuss our results in the last Section. In what follows we use notation and conventions described in [18].

\section{The $3 \times 3$ Equations}

It has been shown in [18] that the Dirac equation:

$$
\left.\begin{array}{l}
p^{A \dot{B}} \eta_{\dot{B}}=m \xi^{A} \\
p_{A \dot{B}} \xi^{A}=m \eta_{\dot{B}}
\end{array}\right\},
$$

or $\gamma^{\mu} p_{\mu} \Psi=m \Psi, \Psi=(\xi, \eta)^{T}$ with $\gamma^{\mu}$ matrices in spinor representation, can be splitted into two $3 \times 3$ subequations:

$$
\left.\begin{array}{rl}
p^{1 \dot{1}} \eta_{\dot{1}} & =m \psi_{\dot{1}}^{1 \mathrm{i}} \\
p^{2 \dot{1}} \eta_{\dot{i}} & =m \psi_{\dot{1}}^{2 \mathrm{i}} \\
p^{2 \dot{2}} \psi_{\dot{i}}^{1 \dot{1}}-p^{1 \dot{2}} \psi_{\dot{1}}^{2 \dot{1}} & =m \eta_{\dot{i}}
\end{array}\right\},
$$

cf. Eqns. (33) and (34) in [18], provided that the identities:

$$
\begin{aligned}
& \psi_{\dot{1}}^{1 \dot{1}}+\psi_{\dot{2}}^{1 \dot{2}}=\xi^{1}, \\
& \psi_{\dot{1}}^{2 \dot{1}}+\psi_{\dot{2}}^{2 \dot{2}}=\xi^{2},
\end{aligned}
$$

are fulfilled. It should be stressed that (2) and (3) can be written in covariant form as Dirac equations with some projection operators due to identities $p^{2 \dot{1}} \psi^{1 \dot{1}}=p^{1 \dot{1}} \psi^{2 \dot{1}}, p^{2 \dot{2}} \psi^{1 \dot{2}}=$ $p^{1 \dot{2}} \psi^{2 \dot{2}}[18]$.

There is also alternative splitting:

$$
\left.\begin{array}{rl}
p^{1 \dot{1}} \theta_{1 \dot{1}}^{1}+p^{1 \dot{2}} \theta_{1 \dot{2}}^{1} & =m \xi^{1} \\
p_{1 \dot{i}} \xi^{1} & =m \theta_{1 \dot{1}}^{1} \\
p_{1 \dot{2}} \xi^{1} & =m \theta_{1 \dot{2}}^{1}
\end{array}\right\},
$$


with identities involving spinor $\eta_{\dot{A}}$ :

$$
\begin{aligned}
& \theta_{1 \dot{1}}^{1}+\theta_{1 \dot{1}}^{2}=\eta_{\dot{i}}, \\
& \theta_{1 \dot{2}}^{1}+\theta_{2 \dot{2}}^{2}=\eta_{\dot{2}} .
\end{aligned}
$$

Note that some components of spinors $\psi_{\dot{C}}^{A \dot{B}}$ and $\theta_{B \dot{C}}^{A}$ are missing in (2), (3) and (6), (7). We shall address this problem in Section 4.

\section{The 3 × 3 Equations and the Melosh Transformation}

In this Section we show that from the $3 \times 3$ equations (2) and (3) the Melosh form of the Dirac equation follows.

Unitary non-local transformations of the Dirac equation, performed to elucidate its physical meaning, have a long history. The most known is the Foldy-Wouthuysen (FW) transformation [20, 21]. Another important transformation was introduced by Melosh [22]. This transformation, also known as the Wigner-Melosh rotation, was used to interpret deepinelastic scattering within the quark model [22-24]. The Melosh transformed Dirac equation reads:

$$
p_{0} \Psi=\left(\alpha^{3} p^{3}+\beta \sqrt{p_{\perp}^{2}+m^{2}}\right) \Psi
$$

where $p_{\perp}=\left(p^{1}, p^{2}\right)$ and $\Psi=\left(\Psi_{1}, \Psi_{2}, \Psi_{3}, \Psi_{4}\right)^{T}$.

Since there are several matrices commuting with the Melosh hamiltonian there are several possibilities of projecting it onto two component subspaces. For example, applying projection operatos $P_{ \pm}=\frac{1}{2}\left(1 \pm i \alpha^{1} \alpha^{2}\right)$ we get in the spinor representation of Dirac matrices:

$$
\begin{aligned}
& p_{0} \Psi_{A}=\left(-\sigma^{3} p^{3}+\sigma^{1} \sqrt{p_{\perp}^{2}+m^{2}}\right) \Psi_{A}, \\
& p_{0} \Psi_{B}=\left(+\sigma^{3} p^{3}+\sigma^{1} \sqrt{p_{\perp}^{2}+m^{2}}\right) \Psi_{B},
\end{aligned}
$$

where $\Psi_{A}=\left(\Psi_{2}, \Psi_{4}\right)^{T}$ and $\Psi_{B}=\left(\Psi_{1}, \Psi_{3}\right)^{T}$.

To show the connection with our theory we substitute $\psi_{\dot{1}}^{2 \mathrm{i}}$ computed from the second of (2) to the third one to get:

$$
\left.\begin{array}{rl}
p^{1 \dot{1}} \eta_{\dot{i}} & =m \psi_{\dot{i}}^{1 \dot{1}} \\
p^{2 \dot{2}} \psi_{\dot{i}}^{1 \dot{1}} & =m\left(1+\frac{p^{1 \dot{2}} p^{2 \mathrm{i}}}{m^{2}}\right) \eta_{\dot{i}}
\end{array}\right\} .
$$

After a non-local substitution, $\sqrt{1+\frac{p^{12} p^{2 i}}{m^{2}}} \eta_{i}=\varphi$, we obtain equation:

$$
\left.\begin{array}{rl}
p^{1 \dot{1}} \varphi & =m \sqrt{1+\frac{p^{12} p^{2 i}}{m^{2}}} \psi_{\dot{i}}^{1 \dot{1}} \\
p^{2 \dot{2}} \psi_{\dot{i}}^{1 \dot{1}} & =m \sqrt{1+\frac{p^{12} p^{2 i}}{m^{2}}} \varphi
\end{array}\right\},
$$


or

$$
p^{0} \Phi_{A}=\left(-\sigma^{3} p^{3}+\sigma^{1} \sqrt{m^{2}+p_{\perp}^{2}}\right) \Phi_{A} .
$$

where $\Phi_{A}=\left(\varphi, \psi_{j}^{1 \mathrm{i}}\right)^{T}$.

It follows that, starting from (2), we have obtained the first Melosh equation (11) for the wavefunction $\Phi_{A}$. Analogously, the second Melosh equation (12) follows easily from (3). The procedure described above bears some analogy to the lightlike reduction of the $(3+1)$-dimensional massless Dirac equation yielding the Levy-Leblond equation in $(2+1)$ dimensions, which can be further splitted into two two-component equations [25].

\section{Mechanism of Fermion-Boson Transition}

Let us demand that spinors $\psi_{\dot{C}}^{A \dot{B}}, \eta_{\dot{A}}(x)$ can be expressed as:

$$
\eta_{\dot{A}}(x)=\chi(x) \hat{\alpha}_{\dot{A}}, \quad \psi_{\dot{C}}^{A \dot{B}}(x)=\psi^{A \dot{B}}(x) \hat{\alpha}_{\dot{C}},
$$

where $\hat{\alpha}_{\dot{A}}$ is a constant spinor (i.e. independent on $x$ ). Substituting (16) into (2) and (3) we get:

$$
\begin{aligned}
& p^{1 \mathrm{i}} \chi(x) \hat{\alpha}_{\mathrm{i}}=m \psi^{1 \mathrm{i}}(x) \hat{\alpha}_{\mathrm{i}} \\
& \left.p^{2 \mathrm{i}} \chi(x) \hat{\alpha}_{\mathrm{i}}=m \psi^{2 \mathrm{i}}(x) \hat{\alpha}_{\mathrm{i}}\right\} \text {, } \\
& \left.p^{2 \dot{2}} \psi^{1 \dot{1}}(x) \hat{\alpha}_{i}-p^{1 \dot{2}} \psi^{2 \dot{1}}(x) \hat{\alpha}_{i}=m \chi(x) \hat{\alpha}_{i}\right\} \text {, } \\
& p^{1 \dot{2}} \chi(x) \hat{\alpha}_{\dot{2}}=m \psi^{1 \dot{2}}(x) \hat{\alpha}_{\dot{2}} \\
& \left.p^{2 \dot{2}} \chi(x) \hat{\alpha}_{\dot{2}}=m \psi^{2 \dot{2}}(x) \hat{\alpha}_{\dot{2}}\right\} \text {. } \\
& -p^{2 \dot{\mathrm{i}}} \psi^{1 \dot{2}}(x) \hat{\alpha}_{\dot{2}}+p^{1 \dot{\mathrm{i}}} \psi^{2 \dot{2}}(x) \hat{\alpha}_{\dot{2}}=m \chi(x) \hat{\alpha}_{\dot{2}}
\end{aligned}
$$

Equations (17) and (18) are proportional to $\hat{\alpha}_{1}, \hat{\alpha}_{\dot{2}}$, respectively. Therefore, these equations are obviously fulfilled if the following $3 \times 3$ equations, obtained by neglecting factors $\hat{\alpha}_{i}, \hat{\alpha}_{\dot{2}}$ :

$$
\left.\begin{array}{rl}
p^{1 \mathrm{i}} \chi(x) & =m \psi^{1 \mathrm{i}}(x) \\
p^{2 \mathrm{i}} \chi(x) & =m \psi^{2 \mathrm{i}}(x) \\
p^{2 \dot{2}} \psi^{1 \mathrm{i}}(x)-p^{1 \dot{2}} \psi^{2 \dot{1}}(x) & =m \chi(x) \\
p^{1 \dot{2}} \chi(x) & =m \psi^{1 \dot{2}}(x) \\
p^{2 \dot{2}} \chi(x) & =m \psi^{2 \dot{2}}(x) \\
-p^{2 \dot{\mathrm{i}}} \psi^{1 \dot{2}}(x)+p^{1 \dot{\mathrm{i}}^{2}} \psi^{2 \dot{2}}(x) & =m \chi(x)
\end{array}\right\},
$$

hold. It follows that (19) and (20) are the $3 \times 3$ equations obtained by splitting the DKP equation, cf. Eqns. (12) and (13) in [18]. Moreover, the problem mentioned at the end of Section 2 is solved due to ansatz (16) -all components of spinors $\psi^{A \dot{B}}, \hat{\alpha}_{\dot{A}}$ are present in (17) and (18). Equations (19) and (20) can be also written as the Dirac equations with some projection operators [18].

Components of the $\psi_{\dot{D}}^{B \dot{C}}$ have still to fulfill the identities (4) and (5). Substituting the ansatz (16) into (4) and (5) and taking into account equations (17) and (18) we arrive at consistency conditions:

$$
\begin{aligned}
& p^{1 \dot{1}} \chi(x) \hat{\alpha}_{\dot{1}}+p^{1 \dot{2}} \chi(x) \hat{\alpha}_{\dot{2}}=\xi^{1}(x), \\
& p^{2 \dot{1}} \chi(x) \hat{\alpha}_{\dot{1}}+p^{2 \dot{2}} \chi(x) \hat{\alpha}_{\dot{2}}=\xi^{2}(x),
\end{aligned}
$$


or $p^{A \dot{B}} \chi(x) \hat{\alpha}_{\dot{B}}=\xi^{A}(x)$, which expresses spinor $\xi^{A}(x)$ in terms of the scalar $\chi(x)$ and a constant spinor $\hat{\alpha}_{\dot{A}}$.

\section{Discussion}

We have shown that it is possible, starting with the Dirac equation (1), to obtain the following chain of equations:

1. splitted Dirac equations (2) and (3),

2. transformed splitted Dirac equations (17) and (18),

3. (19) and (20) (i.e. splitted DKP Eqns. (12) and (13) of Ref. [18]),

4. and, finally, the spin-0 DKP equation (5) of Ref. [18].

Moreover, solutions of the Dirac equation are transformed into solutions of the DKP equation, provided that the ansatz (16) and conditions (21) and (22) hold. The ansatz reduces also number of spinor components so that (17) and (18) contain all components of spinors $\psi^{A \dot{B}}, \hat{\alpha}_{\dot{A}}$. Let us also notice that although $3 \times 3$ equations can be written in covariant form as Dirac equations with some projection operators [18] both are needed to ensure Lorentz invariance.

It follows that in order to perform the fermion-boson transformation, described in Section 4 , we have to postulate existence of a constant spinor $\hat{\alpha}_{\dot{A}}$. In alternative formulation based on (6-9) we have to introduce a constant spinor $\hat{\beta}^{A}$ (we put in this case $\left.\theta_{B \dot{C}}^{A}(x)=\theta_{B \dot{C}}(x) \hat{\beta}^{A}, \xi^{A}(x)=\chi(x) \hat{\beta}^{A}\right)$. Existence of constant Grassmann spinors (i.e. with anticommuting components) is postulated in supersymmetry [26]. Assuming now that $\hat{\alpha}_{\dot{A}}$ is the Grassmann spinor we arrive at a suggestion of supersymmetry. Of course, the problem of connection of the present formalism with supersymmetry needs further work.

The Melosh rotation has been used to understand better the results of deep inelastic scattering which probes the light-cone (current) quarks rather than the constituent quarks defined in rest frame of the nucleon [22-24]. The Melosh rotation transforms the constituent quark picture to the light-cone formalism. Since the Melosh transformed Dirac equation arises naturally in our theory it seems that fermion-boson transformation described in Section 4 applies to the deep inelasting scattering setting.

Open Access This article is distributed under the terms of the Creative Commons Attribution License which permits any use, distribution, and reproduction in any medium, provided the original author(s) and the source are credited.

\section{References}

1. Jackiw, R., Nair, V.P.: Relativistic wave equation for anyons. Phys. Rev. D 43, 1933 (1991)

2. Plyushchay, M.S.: Relativistic particle with torsion, Majorana equation and fractional spin. Phys. Lett. B 273, 250 (1991)

3. Horváthy, P.A., Plyushchay, M.S., Valenzuela, M.: Bosons, fermions and anyons in the plane, and supersymmetry. Ann. Phys. 325, 1931 (2010)

4. Horváthy, P.A., Plyushchay, M.S., Valenzuela, M.: Supersymmetry between Jackiw-Nair and DiracMajorana anyons. Phys. Rev. D 81, 127701 (2010)

5. Horváthy, P.A., Plyushchay, M.S., Valenzuela, M.: Supersymmetry of the planar Dirac-Deser-JackiwTempleton system and of its nonrelativistic limit. J. Math. Phys. 51, 092108 (2010) 
6. Horváthy, P.A., Plyushchay, M.S., Valenzuela, M.: Bosonized supersymmetry from the Majorana-DiracStaunton theory and massive higher-spin fields. Phys. Rev. D 77, 025017 (2008)

7. Foldy, L.L.: Synthesis of covariant particle equations. Phys. Rev. 102, 568 (1956)

8. Garbaczewski, P.: Boson-Fermion duality in four dimensions: comments on the paper of Luther and Schotte. Int. J. Theor. Phys. 25, 1193 (1986)

9. Simulik, V.M., Krivsky, I.Y.u.: Bosonic symmetries of the massless Dirac equation. Adv. Appl. Clifford Al. 8, 69 (1998)

10. Red'kov, V.M., Tokarevskaya, N.G., Spix, G.J.: Majorana-Oppenheimer Approach to Maxwell Electrodynamics. Part I. Minkowski Space. Adv. Appl. Clifford Al. 22, 1129 (2012)

11. Białynicki-Birula, I., Białynicka-Birula, Z.: The role of the Riemann-Silberstein vector in classical and quantum theories of electromagnetism. J. Phys. A: Math. Theor. 46, 053001 (2013)

12. Okniński, A.: Some remarks on relativistic zero-mass wave equations and supersymmetry. arXiv:1304.2002 [math-ph] (2013)

13. Polyakov, A.M.: Fermi-Bose transmutations induced by gauge fields. Mod. Phys. Lett. A 3, 325 (1988)

14. Simulik, V.M., Krivsky, I.Y.u.: Bosonic symmetries of the Dirac equation. Phys. Lett. A 375, 2479 (2011)

15. Simulik, V.M., Krivsky, I.Yu., Lamer, I.L.: Some statistical aspects of the spinor field Fermi-Bose duality. Cond. Matter Phys. 15, 43101 (2012)

16. Sallhofer, H.: Elementary derivation of the dirac equation (In German). Z. Naturforsch. 33a, 1378 (1978)

17. Sallhofer, H.: Elementary derivation of the Dirac equation. X. Z. Naturforsch. 41a, 468 (1986)

18. Okniński, A.: Supersymmetric content of the Dirac and Duffin-Kemmer-Petiau equations. Int. J. Theor. Phys. 50, 729 (2011)

19. Okniński, A.: Duffin-Kemmer-Petiau and Dirac Equations-A Supersymmetric Connection. Symmetry 4, 427 (2012)

20. Foldy, L.L., Wouthuysen, S.A.: On the Dirac theory of spin 1/2 particles and its non-relativistic limit. Phys. Rev. 78, 29 (1950)

21. De Vries, E.: Foldy-Wouthuysen transformations and related problems. Fort. Phys. 18, 149 (1970)

22. Melosh, H.J.: Quarks: currents and constituents. Phys. Rev. D 9, 1095 (1974)

23. Schlumpf, F.: Relativistic constituent quark model of electroweak properties of baryons. Phys. Rev. D 47, 4114 (1993)

24. Xinyu, Z., Ma, B.-Q.: Proton spin in a light-cone chiral quark model. Phys. Rev. D 85, 114048 (2012)

25. Duval, C., Horváthy, P.A., Palla, L.: Spinors in non-relativistic Chern-Simons electrodynamics. Ann. Phys. 249, 265 (1996)

26. Signer, A.: ABC of SUSY. J. Phys. G: Nucl. Part. Phys. 36, 073002 (2009) 